\title{
A damage detection system using modulated ultrasonic and fiber Bragg grating
}

\author{
Xiao Huang ${ }^{1, a}$ and Yuegang Tan ${ }^{1, b}$ \\ ${ }^{1}$ School of Mechanical and Electrical Engineering, Wuhan University of Technology, 122 Luoshi \\ Road, Wuhan, Hubei, P.R.China \\ aemail:51277619@qq.com, bemail:ygtan@whut.edu.cn
}

Keywords: modulated ultrasonic; fiber Bragg grating; damage detection

Abstract. This study presents a damage detection system using modulated ultrasonic and fiber Bragg grating (FBG) in Aluminum thin plate. In the system, modulated ultrasonic technology and FBG sensing technology were combined to detect damage. Piezoelectric patches used to excite ultrasonic and FBG were pasted on a Acrylic plate and then contacted with specimen by coupling, so the excitation point of ultrasonic and sensing point of FBG can be modified arbitrarily. Experiment results showed that the system can detect the damage signal more flexibly.

\section{Introduction}

Ultrasonic techniques have gained prominence for damage detection. The linear property modification of ultrasonic waves, such as reflection, attenuation, mode conversion etc, are utilized in conventional techniques. However, these linear features are not sensitive enough for micro damage $^{(1,2)}$.Modulated ultrasonic technology is rapidly developed during last several years becuase it is more sensitive to micro damage than linear ultrasonic. ${ }^{(3,4,5,6)}$.

In recent years, fiber Bragg grating (FBG) has been expected to be a valuable alternative of ultrasonic detector. FBG posses several advantages over the conventional electrical sensors, such as resistance to electromagnetic interference, high reliability, low cost and so on ${ }^{(7,8,9,10)}$.

In the paper, the modulated ultrasonic technology and FBG technology were combined. In order to make the detecting system more flexible and simpler, ultrasonic waves were both generated by creative mobile piezoelectric patches (MPP), and the response nonlinear signal was detected by a mobile FBG. The structure of mobile detecting system was introduced. Furthermore, the effect of relative location of excitation points, crack and FBG was discussed.

\section{Principle of modulated ultrasonic}

When vibration wave and ultrasonic wave, at distinctive frequencies $f_{1}$ and $f_{2}\left(f_{1}<20 \mathrm{kHz}, f_{2}>20 \mathrm{kHz}\right)$, propagate through a crack in a plate-like structure in the $z$-direction, the total particle displacement $u^{T}$ can be written as follows ${ }^{(11)}$ :

$$
u^{T}=u^{(1)}+u^{(2)}+u^{(3)}
$$

where, $\mathrm{u}^{(1)}$ is linear response, $\mathrm{u}^{(2)}$ is harmonics, and $\mathrm{u}^{(3)}$ is modulation.

$$
\begin{aligned}
& u^{(1)}=u_{1} e^{i\left(k_{1} z-2 \pi f_{1} t+\theta_{1}\right)}+u_{2} e^{i\left(k_{2} z-2 \pi f_{2} t+\theta_{2}\right)} \\
& u^{(2)}=u_{21} e^{2 i\left(k_{1} z-2 \pi f_{1} t+\theta_{1}\right)}+u_{22} e^{2 i\left(k_{2} z-2 \pi f_{2} t+\theta_{2}\right)} \\
& u^{(3)}=u_{m} e^{i\left[\left(k_{2} \pm k_{1}\right) z-2 \pi\left(f_{2} \pm f_{1}\right) t+\left(\theta_{2} \pm \theta_{1}\right)\right]}
\end{aligned}
$$

where $u_{1}$ and $u_{2}$ are the amplitudes of the linear wave at $f_{1}$ and $f_{2}, u_{21}$ and $u_{22}$ are the amplitudes of the nonlinear harmonics at $2 f_{1}$ and $2 f_{2}$, and $u_{m}$ is the amplitude of the modulation responses at $f_{2} \pm f_{1}$ which 
are called sideband. $k_{1}$ and $k_{2}, \theta_{1}$ and $\theta_{2}$ are the wavenumbers and phases respectively. Here, higher-order harmonics and modulations are ignored for simplicity.

\section{Detecting system demonstrations}

A $400 \times 400 \times 1 \mathrm{~mm}$ aluminum plate with a crack $20 \mathrm{~mm}$ long was used as test specimen. Ultrasonic $\left(f_{2}=111 \mathrm{kHz}, \mathrm{A}_{2}=20 \mathrm{~V}\right)$ and vibration waves $\left(f_{2}=18 \mathrm{kHz}, \mathrm{A}_{1}=50 \mathrm{~V}\right)$ were both excited by signal generator and piezoelectric patches $(1 \mathrm{~mm} \times 0.5 \mathrm{~mm}$, material PIC155). A FBG was pasted on a acrylic plate $(50 \times 20 \times 0.2 \mathrm{~mm})$. A FBG with $5 \mathrm{~mm}$ grating was glued on a $50 \times 20 \times 0.2 \mathrm{~mm}$ acrylic plate and piezoelectric patch on a $50 \times 20 \times 1 \mathrm{~mm}$ acrylic plate. The structures of mobile piezoelectric patch (MPP) and mobile FBG are shown in Fig.1 and Fig.2 respectively.
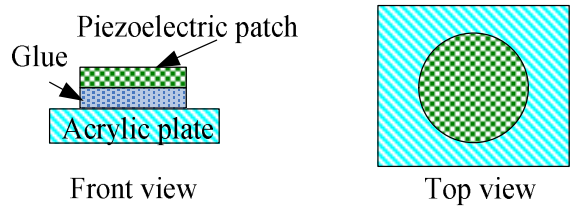

Fig.1. Structure of mobile piezoelectric patch

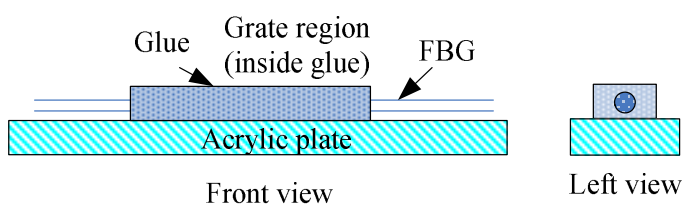

Fig.2. Structure of mobile FBG

The principle of ultrasonic detection using FBG has been described in Reference[12]. The detecting system is shown in Fig.3. MPP and mobile FBG contacted with test specimen by ultrasonic detecting couplant.

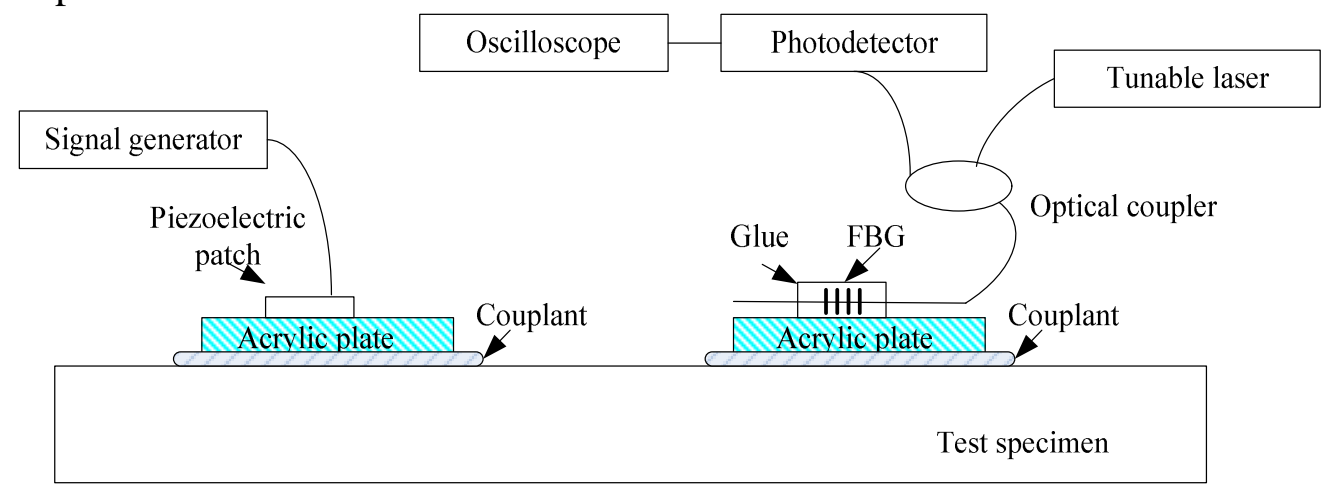

Fig.3. Detecting system

\section{Results and discussion}

In order to ensure the mobile FBG and MPP have good repeatability, four measurements were done, as shown in Fig.4. It can be found the repeatability of four groups of data was good and it will be very helpful to the further research and application.

In order to discuss the interaction of ultrasonic and vibration, vibration signal was fixed at Point $\mathrm{M}$ all the time. Ultrasonic signal was imposed at point A firstly and FBG was located at point S1 and S2 and $\mathrm{S} 3$ respectively to record three data. Then, FBG was located at point S3, and ultrasonic signal was imposed at point $\mathrm{B}, \mathrm{C}$ and $\mathrm{D}$ respectively to obtain the rest three data. $\mathrm{A}_{\mathrm{o}}$ was recorded in Table $1 . \mathrm{Ao}_{1}$ and $\mathrm{Ao}_{2}$ were the frequency spectrum amplitude of response signal at $f_{1}$ and $f_{2}$ respectively. The positions of M, A, B, C, D and S1, S2, S3 were shown in Fig.5 
In Table 1, it was noticed: (1) No matter where the excitation and sensing FBG were, sideband signal can be detected. (2) For the same sensing FBG and vibration point, different ultrasonic excitation point did not influence Ao1,but acted on Ao. (3) The product of Ao1 and $\mathrm{Ao}_{2}$ and crack determined Ao, and it was better to locate ultrasonic and vibration excitation on two sides of crack.

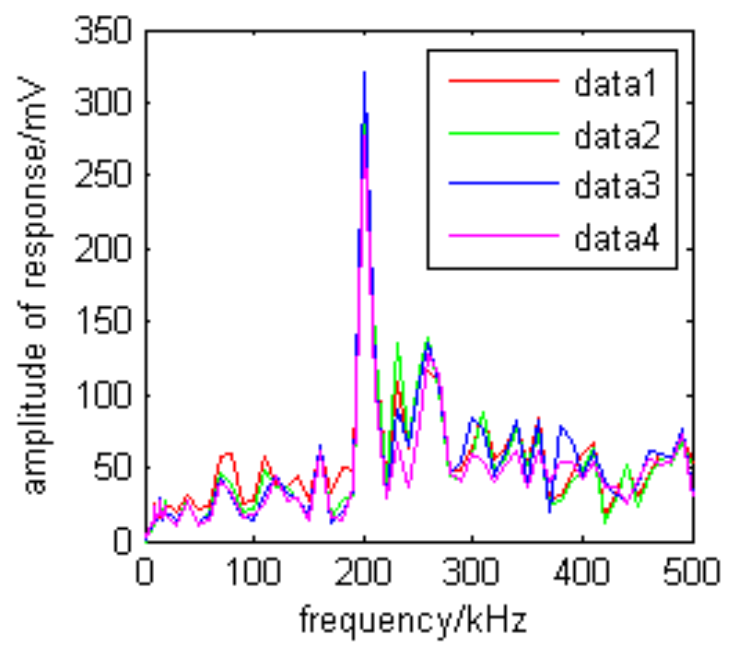

Fig.4. Multi-measurements under MPP excitation

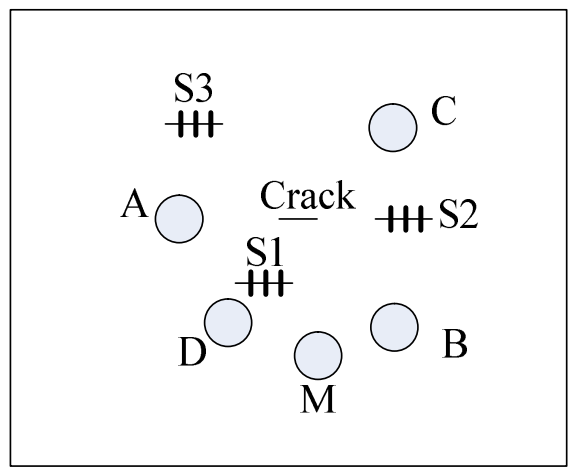

Fig.5. Location point of excitation and FBG

Table 1 Influence of different ultrasonic excitation and sensing FBG

\begin{tabular}{|c|c|c|c|c|c|c|}
\hline $\begin{array}{c}\text { Excitation and sensing } \\
\text { point }\end{array}$ & $\mathrm{A}-\mathrm{S} 1$ & $\mathrm{~A}-\mathrm{S} 2$ & $\mathrm{~A}-\mathrm{S} 3$ & $\mathrm{~B}-\mathrm{S} 3$ & $\mathrm{C}-\mathrm{S} 3$ & $\mathrm{D}-\mathrm{S} 3$ \\
\hline $\mathrm{A}_{\mathrm{o}} / \mathrm{mV}$ & 1.259 & 0.968 & 1.808 & 1.718 & 1.574 & 1.947 \\
\hline $\mathrm{Ao}_{1} / \mathrm{mV}$ & 36.43 & 19.22 & 44.6 & 44.6 & 44.87 & 44.72 \\
\hline $\mathrm{Ao}_{2} / \mathrm{mV}$ & 13.79 & 17.09 & 28.29 & 29.21 & 12.72 & 33.21 \\
\hline $\mathrm{Ao}_{1} \mathrm{Ao}_{2} /(\mathrm{mV})^{2}$ & 502.4 & 328.47 & 1261.73 & 1302.77 & 570.75 & 1487.7 \\
\hline $\mathrm{Ao} /\left(\mathrm{Ao}_{1} \mathrm{Ao}_{2}\right)$ & 0.0025 & 0.03 & 0.0014 & 0.00218 & 0.00276 & 0.0013 \\
\hline
\end{tabular}

\section{Conclusion}

In this study, a detecting system using MPP and mobile FBG was developed. MPP was used for the generation high- frequency ultrasonic wave, and mobile FBG was used to sense the modulated signal. MPP made the system more flexible and made it easier to study the interaction of vibration and ultrasonic. Mobile FBG can be located arbitrarily. In the further research, the system will be applied to obtain many data of nonlinear sideband signal and determine the location of crack.

\section{Acknowledgements}

This work was financially supported by National Natural Science Foundation of China(51375358). 


\section{References}

[1] Hoon Sohn, Hyung Jin Lim, Martin P. DeSimio, Kevin Brown and Mark Derriso. Journal of Sound and Vibration. Vol. 333 (2014),p.1473-1484.

[2] F.C. Campbell. Elements of Metallurgy and Engineering Alloys[M]. ASM International, 2008.

[3] Kyung-Young Jhang. International Journal of Precision Engineering and Manufacturing. Vol.10 (2009), p.123-135.

[4] Tae Hun Lee and Kyung Young Jhang. NDT\&E International. Vol.42 (2009): p.757-764.

[5] Zhongqing Su, Chao Zhou, Ming Hong, Li Cheng, Qiang Wang and Xinlin Qing. Mechanical Systems and Signal Processing. Vol.45 (2014), p.225-239.

[6] Guoshuang Shui, Yue-sheng Wang, Peng Huang and Jianmin Qu. NDT\&E International. Vol.70 (2015), p. 9-15.

[7] Jung-Ryul Lee, Hiroshi Tsuda and Bon-Yong Koo. Optics \& Laser Technology. Vol.39 (2007), p.157-164.

[8] Graham Wild and Steven Hinckley. IEEE SENSORS JOURNAL. Vol.10 (2010), p.805-806.

[9] Tongqing Liu and Ming Han. IEEE SENSORS JOURNAL. Vol.12(2012), p.2368-2373.

[10] Hiroshi Tsuda ,Jung-Rytul Lee, Yisheng Guan, and Junji Takatsubo. Optical Fiber Technology. Vol.13(2007), p. 209-214.

[11] W.J.N. de Lima and M.F. Hamilton. Journal of Sound and Vibration. Vol.265 (2003), p.819-839

[12] Li Cai, Yuegang Tan and Qin Wei. Sensor Review. Vol.35(2015), p.287-295.

[13] Yuegang Tan, Lijun Meng and Dongsheng Zhang. Measurement. Vol.46(2013), p.294-304. 